\title{
A SOA-Based Module for the Production of Geo-Summaries
}

\author{
Elena Roglia, Joint Research Centre, Institute for Environment and Sustainability, Ispra, Italy \\ Rosa Meo, Department of Computer Science, Turin University, Turin, Italy
}

\begin{abstract}
The authors present in this paper the functionalities of the Metadata Retrieval (MDR) module, a software component developed to enrich the information content of a system controlling a fleet of Unmanned Aircraft Vehicles (UAVS) by means of annotations on locations provided by Volunteered Geographical Information (VGI) projects. MDR, developed in the context of an overall project based on SOA architecture, adds updated information to the system via Web Services provided by OpenStreetMap and GeoNames. Furthermore, MDR can be applied for the acquisition and the integration of users'spatial annotations and use them in any way to produce, illustrate and enhance geo-summaries. Actually MDR performs the content characterization of a spatial area by means of a filter that selects the statistically significant annotations whose frequency in the area is in contrast with the surrounding.
\end{abstract}

Keywords: $\quad$ Crowdsourcing, Geo-Summary, Metadata, Statistical Filter, Tags, Volunteered Geographic Information (VGI)

\section{INTRODUCTION}

Natural disasters like floods, landslides and fires require an increasing activity by civil protection agencies involved in territory monitoring. Territory monitoring is needed also in industrial and in intensive agriculture areas in order to ensure protection against the occurrence of water pollution and disposal of waste without a permit. The Advanced Monitoring System of the Territory (SMAT) is a distributed system for the monitoring of the territory that performs territory surveillance using a fleet of different Unmanned Aircraft Vehicles (UAVs). Each UAV, equipped with proper payload sensors, downloads to its ground control station (CS) video and images of the target territory. In turn, each ground control station communicates to the central Supervision and Coordination Station (SS\&C) the received data in near-real-time. The SS\&C performs data storage and near-real-time data fusion.

The software component described in this paper is one of the geo-spatial services provided by SS\&C and is called Metadata Retrieval (MDR). MDR has been developed to enrich and enhance the information collected by UAVs' missions and the information provided by authoritative geospatial data with data coming from Volunteered Geographical Information projects.

The presence of abundant structured and social data on the Web is providing new opportunities of searching for information. 
The ability of Web 2.0 applications to solicit feedback from crowds enables a new class of applications on the Web, which consist in methods for enhancing data and solving tasks by soliciting the contribution from the crowds. The resulting data are later aggregated, analyzed and used to solve some complex task, or they are published in a structured and semantic form on the Web performing the so-called mashup applications. VGI is closely related to the concept of crowdsourcing with the notion that information obtained from a crowd of many observers is likely to be closer to the truth than information obtained from one observer (Gooldchid, 2010).

Authoritative geospatial data are full of detailed information, but can be outdated and frequently very costly. Furthermore, they often do not contain all the information that is needed by final user. On the contrary, in Internet, a large amount of geo-referenced data is generated by the VGI projects through the everyday experience of people. This information is constantly updated by people that use handhelds, pocket PCs or mobile phones connected to the Web. For this reason we decided to use it to increase the information base of the SS\&C database. Information from Internet is used to provide additional information related to the locations monitored by means of UAVs, without in any way replacing the valuable information collected by the UAVs payload sensors but integrating it in the resulting interactive map performing a sort of mashup.

Information on locations provided by VGIs will be henceforth referred as metadata.

Metadata are extracted from several XMLbased (Bray, Paoli, Sperberg-McQueen, Maler \& Yergeau, 1997) files, downloaded from the Web through Web Services provided by open, collaborative projects like OpenStreetMap (www.openstreetmap.org) and GeoNames (www.geonames.org). XML files are checked by the MDR system to be in a well-formed format. Metadata are also cleaned for the possible presence of duplicates and errors.

Finally, metadata can be used to produce a geo-summary of the target area. The geo-summary is composed by annotations filtered from the amount of metadata downloaded from the Web. The selection of the features of the geo-summary occurs by a statistical test, to guarantee statistical significance and as such, an increased level of reliability. The test aims to identify the typical features of a target area. The typical features are those that in the target area are surprising because in contrast to the surroundings.

\section{PREVIOUS WORK}

There are many Geospatial Web Services (GWS) available on the Web. These Web technologies are used to manage, analyse and distribute spatial information (Zhao, Yu $\&$ Di, 2006). The standards proposed by the Open Geospatial Consortium (OGC), through a participatory process, form a solid basis for developing GWS and most of them are used in the MDR module. The concept of Web Service implies an architectural model made of distributed objects or applications, which are located in different parts of the network and/ or different platforms. By taking advantage of the Web Services technology, the SMAT system is implemented according to Service Oriented Architecture (SOA) paradigm.

Web Services are also widely used in the development of a Spatial Data Infrastructure (SDI). SDI denotes the relevant base collection of technologies and policies that facilitate the availability and access to spatial data. It provides a basis for spatial data discovery, evaluation, and application for users and providers (GSDI, 2004). Due to the size of the organizations, one of the principles is that data and metadata are not managed centrally but by the data originator/ owner. In this context, the SMAT system could act the role of a single SDI actor.

Crowdsourcing has emerged as an important paradigm in human problem solving on the Web. It consists in programs that outsource tasks to humans which are difficult to implement automatically in software. In particular, service-oriented crowdsourcing enhances these 
11 more pages are available in the full version of this document, which may be purchased using the "Add to Cart" button on the publisher's webpage: www.igiglobal.com/article/a-soa-based-module-for-the-production-ofgeo-summaries/105089

\title{
Related Content
}

\author{
Insurance-Based Business Web Services Composition \\ An Liu, Liu Wenyin, Liusheng Huang, Qing Li and Mingjun Xiao (2012). Intelligent and \\ Knowledge-Based Computing for Business and Organizational Advancements (pp. \\ 157-173). \\ www.irma-international.org/chapter/insurance-based-business-web-services/65792 \\ Thinking Critically About the Fourth Industrial Revolution as a Wicked \\ Problem \\ Shaun Ruysenaar (2021). Handbook of Research on Using Global Collective \\ Intelligence and Creativity to Solve Wicked Problems (pp. 1-26). \\ www.irma-international.org/chapter/thinking-critically-about-the-fourth-industrial-revolution-as-a- \\ wicked-problem/266776
}

Design Automation, Modeling, Optimization, and Testing of Analog/RF Circuits and Systems by Particle Swarm Optimization Jai Narayan Tripathi, Jayanta Mukherjee and Prakash R. Apte (2013). Swarm Intelligence for Electric and Electronic Engineering (pp. 57-70).

www.irma-international.org/chapter/design-automation-modeling-optimization-testing/72823

An Autonomous Agent Approach to Query Optimization in Stream Grids Saikat Mukherjee, Srinath Srinivasa and Krithi Ramamritham (2012). Intelligent and Knowledge-Based Computing for Business and Organizational Advancements ( $p p$. 276-299).

www.irma-international.org/chapter/autonomous-agent-approach-query-optimization/65799

Computational Techniques to Manage Natural Resources

Diana F. Adamatti and Marilton S. de Aguiar (2012). International Journal of Organizational and Collective Intelligence (pp. 117-137).

www.irma-international.org/article/computational-techniques-to-manage-naturalresources/93079 